\title{
A CONTRIBUTION TO THE PATHOLOGY OF THE SO-CALLED FUNCTIONAL NEUROSES.
}

\author{
By THEO. KLINGMANN, M. D., \\ Pathologist to the Michigan State Asylums for the Insane, University of \\ Michigan, Ann Arbor.
}

(From the Pathological Department of the Michigan State Asylums for the Insane and the Neurological Clinic of the University Hospital.)

Granting that the brain is the organ of the mind and that insanity is a symptom, the result of defective function of this organ, many cases of incipient insanity may be properly classed under this head.

Hysteria, a derangement primarily of the higher cerebral centers and secondarily of the lower centers of the brain and cord, neurasthenia with its endless variety of manifestations, and hypochondriasis-all morbid states of the nervous system, in which there is mental depression, and which are frequently premonitory signs of more profound morbid mental conditions-are symptoms rather than distinct diseases, mere morbid manifestations of a definite pathological process. We must agree that these conditions cannot exist without a change in the material structure of the organs involved.

If we limit pathological research to pathological anatomy, to the post-mortem room and laboratory, we invariably come to the conclusion that certain conditions of the body, in consequence of which some special form of disturbance of the nervous functions may be exhibited, are purely functional. On the other hand, the pathologist who makes his investigations on a broad comprehensive basis, and studies mental and nervous disorders in the broad light of general pathology, making diligent search in every branch of science allied to general medicine, will attain intelligent views as to the real meaning of disturbed nervous functions, and will fully agree that there can be no morbid manifestations with- 
out structural changes of the tissues involved. He will accept the theory that all mental activity has its physiological and anatomical nervous mechanism, and that destruction of this mechanism means destruction of psychological and physiological function.

While we admit that in many instances no gross pathological changes are found in the cerebral cortical tissue, it less frequently happens that the microscope fails to demonstrate intracellular changes. It is true that this is not the primary lesion, but the result, depending on alterations in the nutrition of the nerve elements.

Recent study of a great variety of cases of this class bears out the assumption that disturbances of the gastro-intestinal tract are attended by bacterial necrosis, and the introduction into the general circulation of certain very virulent toxic agents, whose effects are expended largely on the nervous system.

It has been clearly demonstrated that the study of morbid anatomy alone cannot give us all the information that is desired, when the initial cause of the so-called functional disorders is not known. At the most it can only reveal to us the effects of preexisting factors, and leaves us in the dark as regards the primary cause. This fact led the author to consider the subject from another aspect. The conviction is steadily growing in the minds of neurologists that the actual agents which produce tissue changes are toxic substances of one form or another.

Experimental biology has given much aid in studying the action of chemic-toxic substances on cell-protoplasm. That modern biology and general pathology are inseparately associated, has long been recognized, and becomes more and more evident since research with modern comparative experimental methods has been a competent guide for us in the study of special pathology; whence it results that much of the recent advance in the study of cellular pathology must be accredited to experimental biology.

Personal observations made some years ago have led the author to believe that experimental biology is a most valuable adjunct to the study of modern cytology, especially in psychopathology. The results of these studies give us fairly good reason for believing that the earliest changes occurring in the plant 
cell, when treated with various toxins, represent the beginning of death, while they much resemble those found in higher animal life.

For the study of the finer intracellular physiological and pathological changes, especially those artificially produced, we must select cells presenting those conditions which are favorable to, and permit of, observations under a high magnifying power during life. We must first become thoroughly familiar with the morphology and physiology of these cells, before we can state where physiological processes end and pathological changes begin. The most suitable are the cells of the algæ, such as the spirogyra majuscula or laxa. These present a clear, transparent vegetable cell, in which the protoplasmic movements can be carefully observed with a moderately high magnifying power, and which can be preserved in a normal state for a considerable length of time. The forms most appropriate for the study. of structural changes in the animal cell are the lower organisms belonging to the genus of protozoa, the class of rhizopoda, such as the flagellata and ciliata. Extensive observations have also been made on the amœba radiosa and infusoria.

It has been found that a variety of changes occurring in the cells of the spirogyra could be produced by artificial means, three different special forms being distinguished and described as (I) those identical with the physiological appearances occurring in the cells when they gradually die from nature's own causes, and especially those incident to indoor cultivation; (2) those of a purely chemical reaction; (3) a pathological condition produced by toxic substances which Naegeli termed "Oligodynamische Erscheinungen," and which, as the term indicates, he supposed to be a tearing loose of the chlorophyl bands from the protoplasmic cylinder. To bring about these conditions Naegeli used metals such as copper, silver, lead, zinc, and mercury. It was determined that all three phenomena could be produced by the use of the same chemical substance when employed in different degrees of strength, and that these changes were not qualitative but quantitative. The first was brought about with a moderately strong solution ( $1-500,000$ parts), comparatively speaking, as none of the solutions were any stronger than $1-100,000$ parts. The same morphological changes were produced that occur in the cells 
of the spirogyra when they die a natural death or decay from some unknown cause-an observation so frequently noted in the cultivated specimens. The chlorophyl bands lose their color and are broken up into coarse rounded granules. The pyrenoides, with the leukoblasts, form larger or smaller granular masses, and after a time the protoplasmic tube retracts from the cell wall; the nucleus becomes somewhat enlarged and may be slightly pushed to one side or remain in the center.

The second was caused by a stronger solution $(I-100,000)$ by direct chemical action. The whole content of the cell with the protoplasmic tube retracts from the cell wall; the chlorophyl bands alter in form and color without changing their general arrangement, and without separating from the surrounding parts. The protoplasm becomes cloudy and granular; the nucleus, formerly situated in the center of the cell, is pushed aside against the cell wall.

The third form described by Naegeli was produced by a very dilute solution (I-10,000,000 parts); the quantity of the metal present was so small that it could only be detected chemically by first evaporating Io liters of the solution. Here he describes what appeared to him as a retraction or tearing loose of the chlorophyl bands from the protoplasmic tube. With this the nucleus was drawn to one side, the protoplasm became granular, and later (24-36 hours) the protoplasmic tube retracted from the cell wall.

The author determined that in the form which Naegeli described under (3), the chlorophyl bands do not tear loose from the protoplasmic cylinder, but there is a division of the tube into a very delicate inner cylinder, to which the chlorophyl bands are attached, and an outer, thicker portion, which remains attached to the cell wall. Sometimes it was observed that after the division of the protoplasmic cylinder the chlorophyl bands retracted from the inner portion of the divided cylinder, some of the bands remaining attached only by thin threads of colorless slightly granular protoplasm. If entirely separated, they sometimes collected into irregular masses, and their original arrangement could no longer be recognized. Very frequently it occurred that, some hours after the division of the protoplasmic cylinder, the outer part of this tube retracted from the cell wall, leaving the latter 
perfectly clear; at the same time both the inner, delicate and the outer, thicker part of the protoplasmic cylinder could be distinctly seen, and from this it can be positively stated that this division takes place.

The spirogyra majuscula is particularly suitable for the study of intracellular changes; its chlorophyl bands are widely separated and the nucleus with its finer attachment can be distinctly seen; the protoplasmic movements are easily distinguishable, and the process, which terminates in the death of the cell, can be observed under the microscope from beginning to end. A great many other varieties of spirogyra were used. It was found that by putting pure copper foil, which was first thoroughly cleaned, into distilled water (the water having been distilled from a glass retort and all possibility of contamination from foreign substances excluded), and allowing it to remain there for from 12 to 24 hours, complete destruction of the spirogyra cells occurred, when treated with this fluid. A few drops of the water having been taken and placed on a glass slide, a few threads of spirogyra were put into this and carefully observed under a No. 7 Leitz objective. The protoplasmic current was plainly seen to become slower and slower, until it finally ceased, the protoplasm becoming more granular. After a few moments it was noticed that at the margin of the cell the chlorophyl bands retracted from the cell wall, or, to speak more correctly, there was a division of the protoplasmic tube, the inner part retracting from the outer, and carrying with it into the center of the cell the chlorophyl bands, these remaining attached to this delicate membrane. The fine filaments, which held the nucleus suspended in the center of the cell, became relaxed, the nucleus, which at first had appeared slightly oblong, was now round and somewhat enlarged, and could be seen more distinctly because it had become granular. After a time, if the fibers were allowed to remain in the copper water, it was noticed that the outer portion of the protoplasmic tube retracted from the cell wall, leaving the latter perfectly clear. After a separation of the inner membrane from the outer one, there was still a granular appearance of the whole cell, but as soon as the separation of the outer membrane from the cell wall occurred, the latter remained perfectly clear, showing without doubt that an outer membrane lining the cell wall exists, as well as an inner one surrounding the chlorophyl bands and nucleus. 
Other metals-gold, silver, tin, lead, and mercury-were used in preparing the water with similar results, but none proved so satisfactory as copper. These experiments were carefully controlled by putting spirogyra threads into distilled water that had not been treated with the metal, when it was found that the specimens remained unchanged for weeks.

To study the action of toxic agents on animal protoplasm, a very low class of protozoa was selected. The rhizopodæ were very satisfactory. The amœba difflugia oblonga, hæmatococcus pluvialis, paramæzium bursaria, spirostomum ambiguum (Ehrbg.), vorticella microstoma and stylongchia mytilus were carefully observed. In all these cases the experiments ended in the death of these low forms of animal life, and considering the great morphological difference in the well-protected plant cell and the practically nude animal protoplasm, the action of the toxic substance can be regarded as parallel. As soon as the protozoa were brought in contact with the copper water, they became less active, and after a variable period of time (from 2 to 24 hours) all motion ceased; from having had an irregular outline, they now became round; those provided with cilia retracted them. A retraction of the protoplasm from its delicate covering, after the cilia had disappeared, was repeatedly observed in the paramæzium. The protoplasm, which before had been perfectly transparent, now took on a dusty appearance. The experiments with bacteria were very interesting.

Without reviewing in detail the significance of the above experiments, I will relate the results of recent investigations. With a view of throwing more light upon the study of intracellular structure and the earliest pathological changes occurring therein, the author instituted a series of experiments very similar to those just cited. In this series the same material as in the preceding experiments was used (the algæ and protozoa), but instead ot employing a metal as the toxic agent, blood-serum obtained from patients suffering from various forms of so-called functional, nervous and mental disorders was used. I selected cases which were, with few exceptions, apparently instances of true functional neuroses. One cubic centimeter of blood-serum was diluted with I liter of distilled water (the water having been distilled by the process described above). In order to produce slow destruction 
of so delicate a structure as the spirogyra, this alga must be treated with very dilute solutions of the toxic substance.

A filament of spirogyra majuscula was placed on a glass slide, and a few drops of toxic solution having been added, it was then observed under a moderately high magnifying power. At first the protoplasmic streaming could be distinctly seen, but in a very short time (I to 2 minutes) it became slower and slower, and in about Io minutes it had entirely ceased. The fine granules, that were carried along in the stream, were no longer propelled, but were in a constant tremulous motion, until the protoplasmic cylinder began to divide, when all signs of life disappeared. The inner part of the cylinder continued to retract, carrying with it the chlorophyl bands which were attached to it. Beyond a slight change in the color and in the general arrangement, nothing noteworthy occurred in the chlorophyl bands until later. The nucleus, that had been oval, became perfectly round and moved toward the periphery of the cell as the retraction of the inner membrane became more marked. The intranuclear substance became cloudy, and fine granules were seen to collect in masses. When the action of the toxin was allowed to continue, the outer protoplast retracted from the cell wall, leaving the latter perfectly transparent. Now a subdivision of the inner protoplast took place, and with this a separation of the chlorophyl bands into irregular masses lying here and there in different parts of the cell; the nucleus was still distinctly visible, but gradually disintegrated into a reddish-brown mass. To eliminate all possibility of error in the method, or contamination of the distilled water with other substances, a filament of spirogyra was placed in the distilled water, and, after several days, was found to be in a perfectly normal condition. This experiment was repeated several times with the same results; various forms of spirogyra were used, but with the exception of a difference in time the action of the toxin was the same. Very interesting changes were observed in the protozoa by treating the hæmatococcus pluvialis with distilled water containing the toxic serum. The movements of the cilia became markedly slower, and, in consequence, locomotion was also retarded; after a few minutes motion had entirely ceased; the cilia appeared shorter and thicker; the oval body took on a rounded form, a dusty appearance, and became somewhat 
- 526 pathology of so-called functional neuroses [Jan.

swollen. Paramæzium bursaria is very susceptible to the action of toxic substances, and dies rapidly when treated with them. Many other forms were observed with the same general results.

A great variety of pathological conditions were demonstrated in the ganglion cells of the higher animals as the result of injecting toxic serum of varying dilutions; the most remarkable feature was that the form of chromatolysis varied as the strength of the serum. For this purpose the guinea-pig and rabbit were selected. When 2 cubic centimeters of undiluted toxic serum were injected, the animal was at once thrown into convulsions, and in a few minutes death took place. Microscopic examination of the spinal ganglion cells revealed a loss of the peripheral chromatic bodies, while the perinuclear bodies had undergone disintegration. In the cerebral cortex different stages of chromatolysis were noted. In some cells the peripheral chromatic bodies and the dendritic spindles were normal, while in the perinuclear zone there was advanced chromatolysis; in other cells the chromatic substance was absent. If the serum was diluted with ten times its volume of distilled water and injected into the circulation of the guineapig, no convulsions occurred, but after five days of continuous action of the toxin death took place. Upon examination, most of the cells of the cerebral cortex showed peripheral chromatolysis, while no distinct or uniform nuclear changes were detected. Upon further dilution of the toxic serum (I-20) and after daily inoculations, if death did not take place within a reasonable period of time, the animals were killed after the continuous action of the toxin for ten days. In the cerebral cortical cells it was found that the chromatic substance had uniformly disappeared from the periphery; the chromatic bodies seemed to be aggregated in the central portion of the cell, giving the center of the cell a deep-blue appearance, when stained with methylene blue, while the periphery remained unstained. If the animal was allowed to live for 20 days, with daily inoculations of toxic bloodserum, the cortex cells presented a more advanced stage of chromatolysis, a more decided reaction occurred, and in some cells a beginning subdivision of the chromatic substance was observed; at the same time there was a decided change in the nucleus, which was displaced toward the periphery and appeared somewhat enlarged, with alterations in the chromatic portion. 
When the toxic serum was allowed to continue its action for a longer period, the intracellular changes were more advanced, the chromatic substance was subdivided, some of the chromatic bodies were indistinct, and the achromatic substance was chromatophilic. The nuclei contained numerous granules, and the nucleoli were much enlarged. The same experiments were carried out with blood-serum from perfectly healthy individuals, with negative results.

From the results of the experiments detailed above, it is very evident that the cellular changes are quantitative rather than qualitative, and that such an inference is justifiable is very probable from the fact that long-continued action produces more advanced changes. That a toxic condition of the blood exists, and that it is a prominent factor in the etiology of disease is beyond doubt. . Unquestionably many of the cerebral cortical cells are functionally inactive when these marked changes are present.

These experiments led to the establishment of a test for toxic states of the blood, which has been described in another paper. ${ }^{1}$ This has enabled the author to examine a great variety of cases belonging to this class, and others in which bacterial toxins were the cause of the disorder, as in the acute specific infectious diseases, with constant results. It was interesting to note that the action of toxic blood of patients suffering from functional nervous disorders was quite as marked as that of those suffering from acute infectious diseases. It is thereby shown that the disease is not really a product of the structural alterations which may be present, but of a hurtful substance or poison capable of producing these structural changes. When we observe such cases early, we may well say that they are functional neuroses unless we recognize this condition, a toxæmia due to deficient metabolic power and retarded elimination, the injurious effects of which have been demonstrated in the above experiments.

Not only are the higher centers ill-nourished, but they are irretrievably damaged, their working power is annulled, and the whole train of evils follows even to insanity.

A considerable number of the individuals suffering from these maladies are from the outstart handicapped; they have inherited

${ }^{1}$ American Journal of the Medical Sciences, November, 1900. 
528 Pathology of So-Called functional NeUroses [Jan.

a feeble organization, their resistive power is diminished, the functions are perverted most readily, and the entire organism reacts with readiness to these injurious attacks. It is therefore not surprising that an accumulation of a deleterious agent in the nutrient fluid of the body should overstimulate, depress, or destroy the functions of the nervous system, when morbid heredity previously exists. 